\title{
Clinical applications of the MicroPulse diode laser
}

\begin{abstract}
Purpose To evaluate the efficacy of the Iris Oculight MicroPulse $810 \mathrm{~nm}$ diode laser in the treatment of macular oedema secondary to either branch retinal vein occlusion (BRVO) or diabetic maculopathy and in the treatment of proliferative diabetic retinopathy. The specific advantages of this type of laser delivery are greater retinal pigment epithelial specificity and less damage to the inner retina, thus preserving visual field and colour contrast sensitivity.
\end{abstract}

Methods Fifty-two eyes of 33 consecutive patients were treated over a 6 -month period. Thirteen eyes had proliferative diabetic retinopathy and 39 had macular oedema secondary to BRVO or diabetic maculopathy. Panretinal and grid pattern photocoagulation were performed using the micropulse mode with the laser on for 100-300 $\mu$ s and off for between 1900 and $1700 \mu$ s repeatedly in a pulse envelope of $0.1-0.3 \mathrm{~s}$ duration. Microaneurysms were not treated directly. Patients were assessed clinically and angiographically at 3 and 6 months. Results Ten eyes $(77 \%)$ with proliferative disease showed some regression of new vessels at 6 months. Twenty-two eyes (57\%) showed resolution of macular oedema at 6 months. Visual acuity was maintained in $\mathbf{2 7}$ eyes $(69 \%)$ and improved in 11 eyes $(28 \%)$. Conclusion Diode laser in micropulse mode is effective in the management of diabetic and occlusive macular oedema and proliferative diabetic disease.

Key words Diode laser, Micropulse, Photocoagulation

The benefits of laser photocoagulation for the treatment of diabetic retinopathy have been well validated by two major multi-centre randomised prospective clinical trials: the Diabetic Retinopathy Study (DRS) and the Early Treatment of Diabetic Retinopathy Study (ETDRS). The primary effect of laser treatment is a thermal injury induced at the level of the retinal pigment epithelium (RPE), but concurrent damage to adjacent retinal photoreceptors and choriocapillaris occurs as a result of heat transmission. This damage results in loss of central vision, decreased contrast sensitivity and reduced visual fields in a substantial number of patients. ${ }^{1,2}$ Current photocoagulation procedures performed with conventional continuous wave (cw) laser systems cause damage to the neural retinal microstructures by thermal spread from the RPE - a clinically visible burn is taken as the threshold for treatment. It is not possible to isolate the injury to the RPE with cw laser exposure times (50-400 ms); this can only be attempted using much shorter laser exposure times of less than $1 \mathrm{~ms}$ which would confine the damage to the RPE/choroid melanin granules (Fig. 1). It has been shown that laser pulses of extremely short duration affect the RPE alone with little effect on the photoreceptors or choriocapillaris from 'heat flow-away' or thermal spread from the primary irradiated area. This area of heat damage is called the thermal diffusion distance. ${ }^{3}$ As the pulse duration is reduced the radius of the heataffected zone would also be reduced. Table 1 lists the thermal diffusion distance versus pulse duration in water, which is a good

representation for ocular tissue. If the laser 'on' time is shorter than the thermal diffusion time $(100 \mu \mathrm{s})$ the distance between the RPE absorbing melanocytes and the photoreceptors (about $5-10 \mu \mathrm{m}$ from the RPE inner surface) is spared.

This concept of micropulse minimal impact photocoagulation can be achieved using the $810 \mathrm{~nm}$ infrared diode laser. Although dense cataract or significant vitreous haemorrhage reduces transmission of all wavelengths, the diode laser remains effective even when the ocular media are obscured (Table 2). It is well absorbed by the RPE causing a more discrete temperature rise at the inner RPE surface. The diode laser can be electronically controlled to operate in the repetitive micropulse mode and this is a relatively easy technical event. ${ }^{4}$

We wanted to evaluate whether the diode laser in micropulse mode was effective in the treatment of macular oedema secondary to
C.M. Moorman A.M.P. Hamilton Medical Retina Service Moorfields Eye Hospital City Road London EC1V 2PD, UK

Received: 26 May 1998 Accepted in revised form: 1 October 1998 


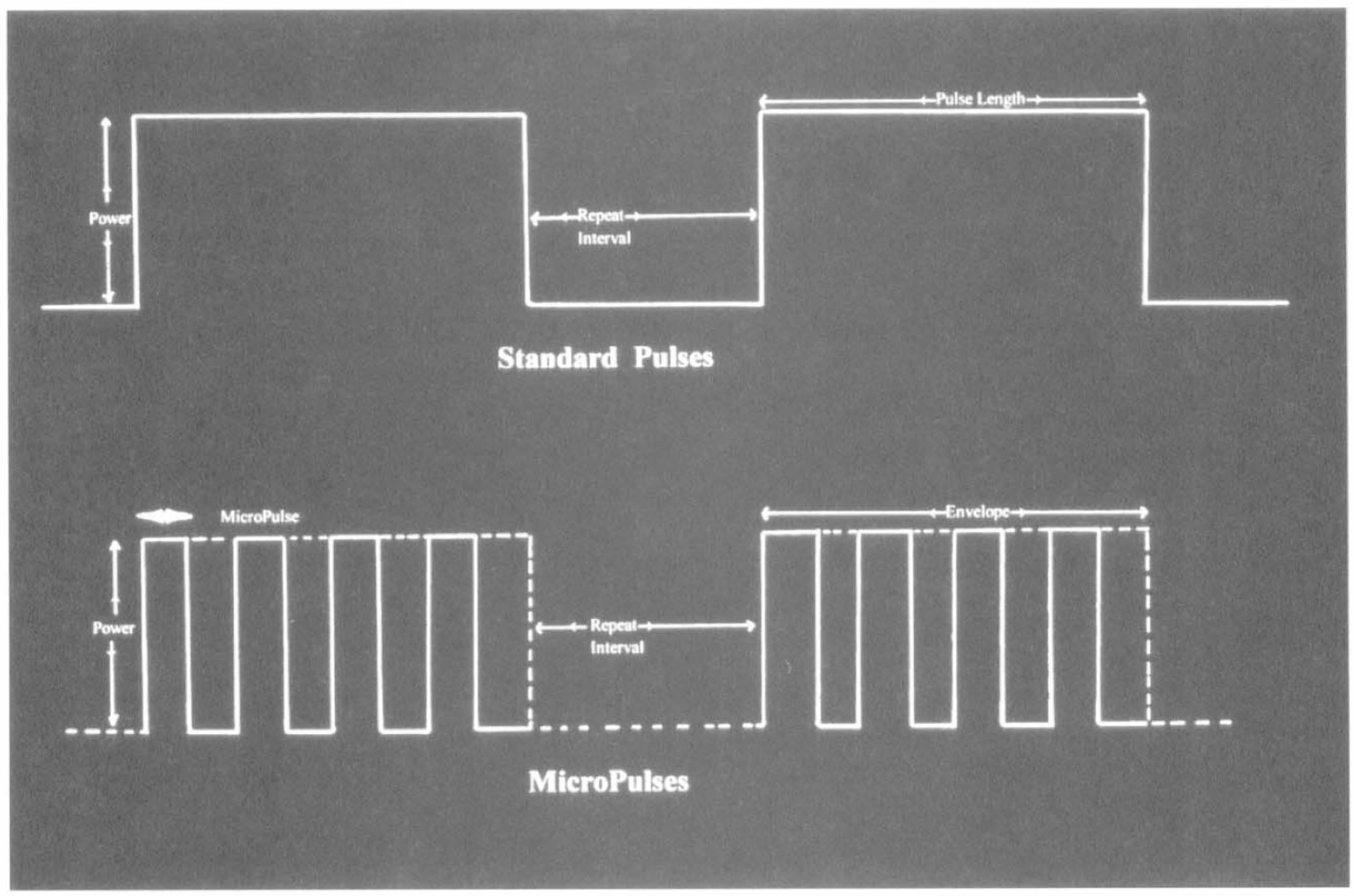

Fig. 1. Diagram to show Standard versus MicroPulse configuration. The pulse length normally indicates the amount of time in milliseconds the laser will deliver the indicated power, either in single-shot or repeat mode. When MicroPulse mode is used the pulse length is an envelope in which one or more smaller pulses may be fired.

branch retinal vein occlusion (BRVO) and diabetic maculopathy and in the treatment of proliferative diabetic retinopathy.

\section{Methods}

Patients attending the Medical Retina Service of Moorfields Eye Hospital over a 6-month period were recruited to the study. To be eligible, patients had to be willing and able to undergo fundus fluorescein angiography and be previously untreated for retinal vascular disorders (Table 3).

All patients were treated by one observer (C.M.M.) using the Iris Oculight Slx MicroPulse diode laser in micropulse mode with the 'on' time set at 100-300 $\mu$ s and the 'off' time set between 1900 and $1700 \mu$ s repeatedly in a pulse envelope of $0.1-0.3 \mathrm{~s}$ duration.

The power was initially adjusted upward to the minimum threshold value for a barely visible burn and then set at half that value during treatment. As the pigment in the RPE changed over the treatment area the visible threshold was checked occasionally.

The spot size used was between 200 and $500 \mu \mathrm{m}$ in diameter. Clinically significant macular oedema was treated with standard grid pattern photocoagulation to areas of retinal thickening; microaneurysms were not specifically targeted. Patients were reviewed clinically and angiographically by two observers at 3 and 6 months and re-treated as necessary. No patient received more than three treatment episodes.

Proliferative diabetic retinopathy was treated with standard panretinal photocoagulation: 1500 burns initially, followed by a further 1500 burns at 6-week intervals depending on the clinical response. Patients were assessed clinically at 3 and 6 months for complete, partial or no regression of new vessels.

All patients were asked to grade the level of discomfort felt during treatment on a scale of 1-10, with 1 representing no sensation at all and 10 representing severe discomfort.

We anticipated the following clinical effects:

1. The damage would be confined to the RPE.

Table 1. Thermal diffusion distance versus pulse duration in water ${ }^{13}$

\begin{tabular}{ll}
\hline Pulse duration & Thermal diffusion distance \\
\hline $1 \mathrm{~s} \mathrm{or} 1000000 \mu \mathrm{s}$ & $1 \mathrm{~mm}$ or $1000 \mu \mathrm{m}$ \\
$0.5 \mathrm{~s}$ or $500000 \mu \mathrm{s}$ & $707 \mu \mathrm{m}$ \\
$100 \mathrm{~ms}$ or $100000 \mu \mathrm{s}$ & $320 \mu \mathrm{m}$ \\
$50 \mathrm{~ms}$ or $50000 \mu \mathrm{s}$ & $225 \mu \mathrm{m}$ \\
$10 \mathrm{~ms}$ or $10000 \mu \mathrm{s}$ & $100 \mu \mathrm{m}$ \\
$1 \mathrm{~ms}$ or $1000 \mu \mathrm{s}$ & $32 \mu \mathrm{m}$ \\
$100 \mu \mathrm{s}^{\mathrm{a}}$ & $10 \mu \mathrm{m}^{\mathrm{b}}$ \\
$10 \mu \mathrm{s}$ & $3.2 \mu \mathrm{m}$ \\
$1 \mu \mathrm{s}$ & $1 \mu \mathrm{m}$ \\
\hline
\end{tabular}

${ }^{2}$ One micropulse.

${ }^{\mathrm{b}}$ Approximate size of a retinal pigment epithelium cell. 
Table 2. Approximate transmission and absorption for laser wavelengths commonly used for retinal photocoagulation

\begin{tabular}{|c|c|c|c|c|c|c|c|}
\hline \multirow[b]{3}{*}{ Laser type } & \multirow[b]{3}{*}{ Wavelength (nm) } & \multicolumn{2}{|c|}{ Ocular media transmission (\%) } & \multicolumn{4}{|c|}{ Retinal chromophore absorption (\%) } \\
\hline & & \multirow[b]{2}{*}{ Young eye } & \multirow[b]{2}{*}{ Old eye } & \multicolumn{2}{|c|}{ Melanin } & \multirow[b]{2}{*}{ Xanthophyll } & \multirow[b]{2}{*}{ Haemoglobin } \\
\hline & & & & RPE + choroid & RPE & & \\
\hline Argon & 488 & 70 & 10 & 70 & 55 & 70 & 70 \\
\hline Dye & 590 & 93 & 80 & 70 & 20 & 2 & 60 \\
\hline Krypton & 647 & 95 & 90 & 60 & 16 & 1 & 15 \\
\hline Diode & 810 & 97 & 95 & 35 & 7 & $<1$ & 8 \\
\hline
\end{tabular}

RPE, retinal pigment epithelium.

2. There would be no thermal effect to the neurosensory retina.

3. There would be no visible ophthalmoscopic endpoint at the time of treatment.

4. There would be little or no patient sensation.

5. There would be no visible light flash so patients should not experience photophobia.

6. The therapeutic effects would be similar to $\mathrm{cw}$ photocoagulation.

\section{Results}

\section{Group 1: Proliferative diabetic retinopathy (Table 4)}

Nine patients were treated for proliferative diabetic retinopathy; 4 patients presented with bilateral disease. Overall the number of burns required to treat new vessels with the diode laser on micropulse mode was between 4500 and 6000 burns per eye (three or four treatment episodes). The average length of time required for patients to respond to treatment was 13 weeks.

At 6 months 8 eyes $(62 \%)$ of 5 patients showed complete regression of new vessels. A further 2 eyes (2 patients) showed partial regression. Both eyes were noted to have extensive ischaemic changes prior to treatment. One of these patients developed cardiac and renal problems during the study period and the other patient had poor glycaemic control throughout; both these factors may have affected the response to treatment. As they had persistent new vessels at the end of the study period they were further treated with argon laser panretinal photocoagulation. Their general medical problems were also being treated and at follow-up 3 months later the vessels had regressed. Three eyes ( 2 patients) showed minimal or no response to laser treatment over the 6-month study period. One patient had new vessels secondary to an ischaemic branch vein occlusion and did not attend regularly for treatment. He received a total of 3233 burns which may simply have been inadequate; he was eventually lost to follow-up.

Table 3. Numbers of eyes/patients treated over 6 months

\begin{tabular}{lcc}
\hline & No. of eyes & No. of patients \\
\hline Proliferative diabetic retinopathy & 13 & 9 \\
Macular oedema & 39 & 24 \\
Total & 52 & 33 \\
\hline
\end{tabular}

The other patient was a young obese woman with bilateral disease who was undergoing fertility treatment. She received 5668 burns to the left eye and 5500 to the right with minimal effect (Fig. 2). In view of her age and lack of response by the end of the study period, she was treated with conventional argon panretinal photocoagulation to both eyes. The new vessels regressed after four treatment sessions and an additional 4500 burns to each eye.

\section{Group 2: Macular oedema (Table 5)}

Thirty-nine eyes of 33 patients were treated for macular oedema. Twenty-two eyes (57\%) demonstrated resolution of macular oedema on angiography at 6 months (Fig. 3). Fifteen eyes remained unchanged on angiography at the end of the study period and 2 eyes (1 patient) were worse. This last was an elderly patient with extensive long-standing macular oedema in both eyes. She had three treatment episodes without effect. Vision improved by 1 or more Snellen lines in 11 patients and was maintained in $27(69 \%)$ patients.

One patient who had treatment for macular oedema returned at 3 months with a vitreous haemorrhage in the treated eye thought to be secondary to disc new vessels; he was removed from the study and treated with argon green panretinal photocoagulation.

There was no difference in response between patients with BRVO and diabetic macular oedema. Over half $(54 \%)$ the eyes treated for macular oedema required more than one treatment episode (Table 6).

\section{Clinical appearance of treated eyes}

There was no visible burn at the time of treatment or immediately afterwards regardless of fundal pigmentation. This meant that the treating doctor had to plan in advance a treatment strategy for panretinal photocoagulation or risk either overtreating or

Table 4. Results of eyes treated for proliferative retinopathy: group 1 $(n=13)$

\begin{tabular}{lccc}
\hline & $\begin{array}{c}\text { Vessels } \\
\text { regressed }\end{array}$ & $\begin{array}{c}\text { Some } \\
\text { regression }\end{array}$ & $\begin{array}{c}\text { No } \\
\text { regression }\end{array}$ \\
\hline At 3 months & $5(39 \%)$ & $3(23 \%)$ & $5(39 \%)$ \\
At 6 months & $8(62 \%)$ & $2(15 \%)$ & $3(23 \%)$ \\
\hline $61.5 \%$ of eyes responded to treatment. & \\
$76.8 \%$ overall showed some regression.
\end{tabular}




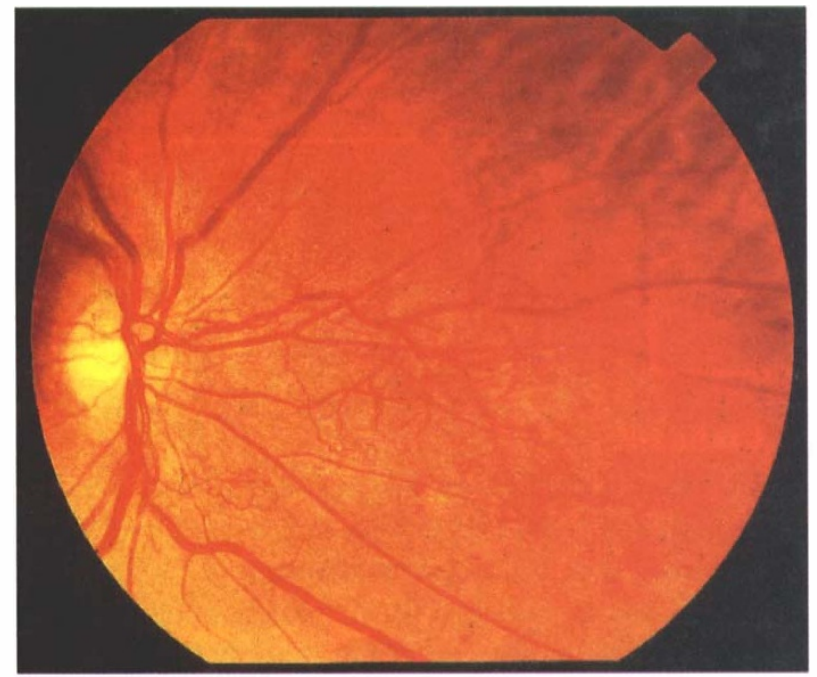

Fig. 2. Patient 1. Right fundus 6 weeks after PRP (3000 burns). New vessels are still present nasally; there are no visible burns.

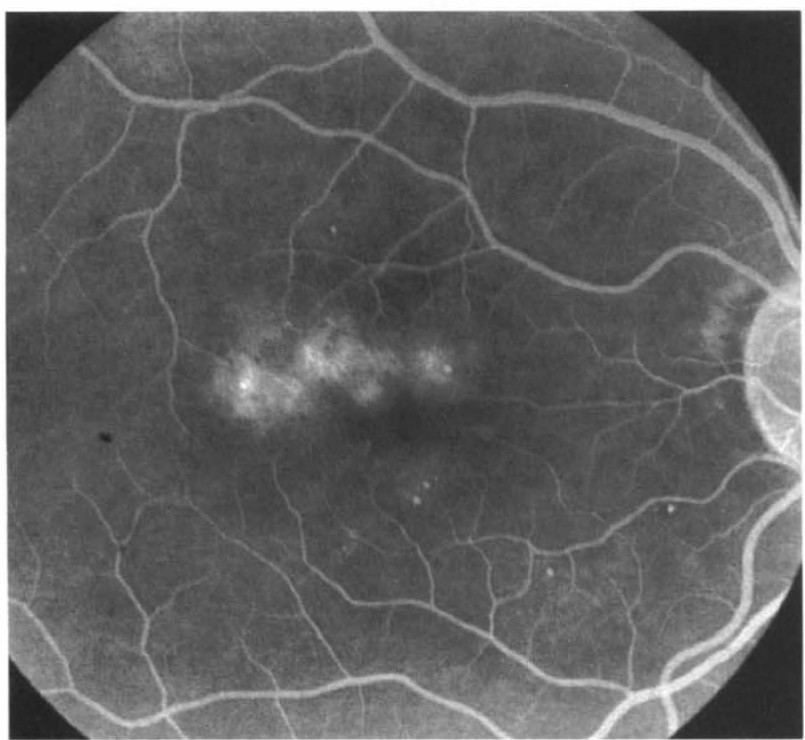

(a)

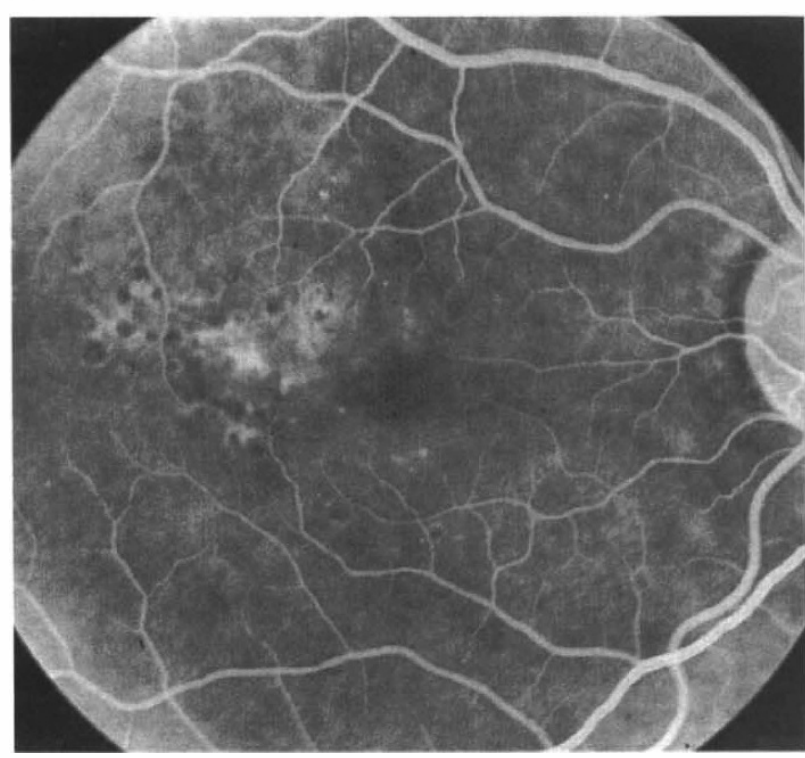

(c)
Table 5. Results of eyes treated for macular oedema: group $2(n=39)$

\begin{tabular}{lccl}
\hline & \multicolumn{3}{c}{ Macular oedema } \\
At 3 months & 20 & Increased & Unchanged \\
At 6 months & $22(57 \%)$ & $2(5 \%)$ & 17 \\
& & Visual acuity & \\
& Improved & Maintained & Worse \\
At 3 months & 5 & 34 & 0 \\
At 6 months & $11(28 \%)$ & $27(69 \%)$ & $1(3 \%)$ \\
\hline
\end{tabular}

$56.5 \%$ of patients showed improvement in macular oedema. $97.0 \%$ of patients maintained or improved their vision at 6 months.

undertreating certain areas. Treatment for eyes with macular oedema was performed with access to the fluorescein angiogram.

At 3 months 8 (62\%) eyes treated with panretinal photocoagulation had developed visible pigmented laser scars; all except 2 were heavily pigmented fundi. One-

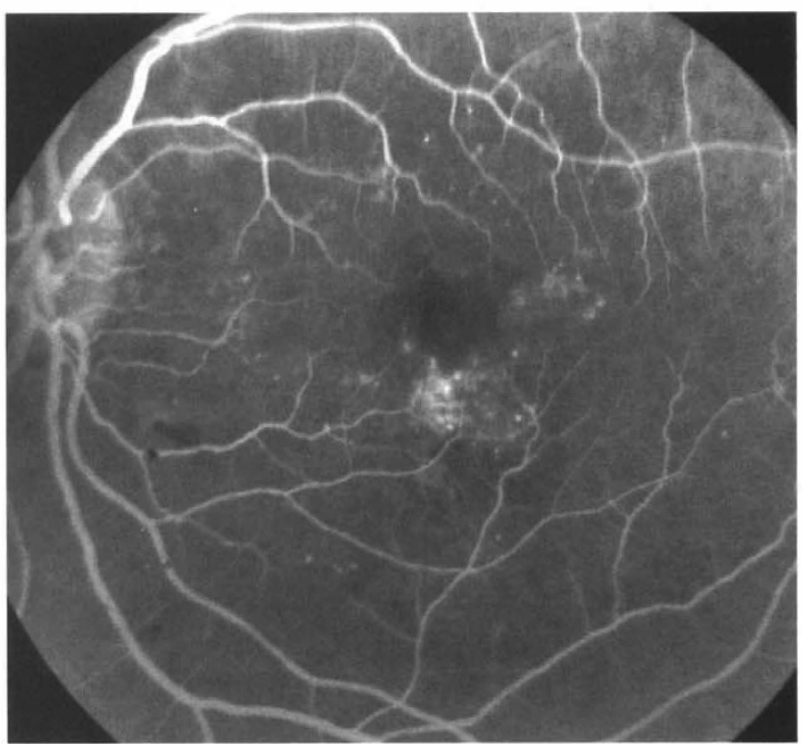

(b)

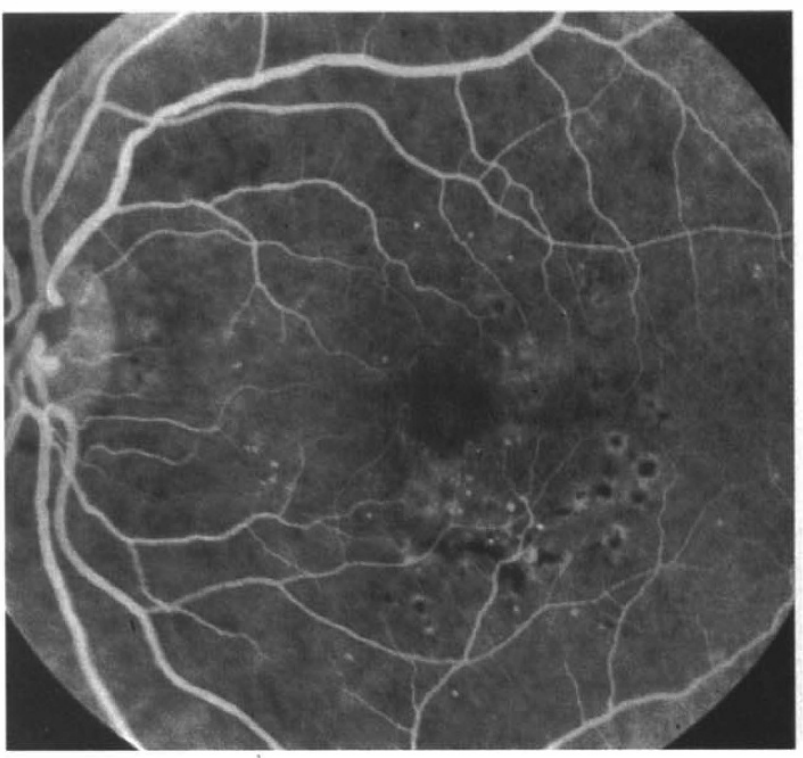

(d)

Fig. 3. (a) Patient 2. Right eye pre-treatment fluorescein angiogram. (b) Left eye pre-treatment fluorescein angiogram. (c) Right eye post-treatment angiogram. (d) Left eye post-treatment angiogram. The macular oedema has resolved. There are no visible burns 3 months after treatment. 
Table 6. Number of treatment episodes per eye for patients treated for macular oedema

\begin{tabular}{lccc}
\hline Episodes: & 1 & 2 & 3 \\
Patients: & 18 & 19 & 2 \\
& $(46 \%)$ & $(49 \%)$ & $(5 \%)$ \\
\hline
\end{tabular}

$54 \%$ of patients required more than one treatment episode.

third (29\%) of eyes treated for macular oedema had faint pigmentary disturbance at the level of the RPE at 3 months (Fig. 4).

By 6 months 12 eyes (35\%) showed no evidence of laser burns even on angiography; of these, 9 eyes had shown clinical and angiographic evidence of resolution of macular oedema.

\section{Patient response to treatment}

Treatment with the diode laser on micropulse mode was extremely well tolerated by all patients. None recorded any sensation during laser treatment (zero on the pain scale). This was especially relevant in patients undergoing panretinal photocoagulation. In addition, as there was no light flash, fixation was good and most patients were able to co-operate well during treatment of the macular region.

\section{Discussion}

The efficacy of both argon and diode laser photocoagulation in the treatment of diabetic maculopathy has been confirmed by the results of several studies. ${ }^{5-7}$ The precise mechanism of action of laser treatment in the resolution of macular oedema remains unclear. It is possible that changes in the microstructure of the retinal pigment epithelial cells result in increased removal of fluid from the retina. What is clear is that direct closure of retinal microaneurysms by heavy treatment with short-wave laser energy is not necessary for fluid resorption. Gentle treatment aimed at the level of the RPE for extremely short periods of time may be just as effective. ${ }^{8}$

Laser treatment of macular oedema with the MicroPulse diode was relatively simple. Patients found it easy to co-operate and were not disturbed by flashing lights, which made fixation more reliable. Certainly our results compare favourably with those achieved using the argon laser. Less than $50 \%$ of patients required retreatment during the 6-month follow-up period. The mechanism of action of panretinal photocoagulation in the treatment of proliferative diabetic retinopathy is also poorly understood. The various hypotheses include the concept of reducing oxygen demands in the outer retina and making choroidal oxygen available to the ischaemic inner retinal layers by creating full-thickness thermal retinal necrosis in areas of non-perfusion. Alternatively it has been suggested that the regeneration and proliferation of new RPE cells, induced by laser photocoagulation, causes the therapeutic effect by restoring the blood-retinal barrier. ${ }^{10}$ In addition, recent studies have found that mild photocoagulation of the RPE may induce the release of vasomodulatory factors that act to inhibit vascular proliferation. ${ }^{11}$

If stimulation of the RPE is all that is required to cause regression of new vessels then a full-thickness burn with all that implies for visual fields and quality of vision would seem unnecessary. In our study satisfactory regression of new vessels was achieved using subthreshold micropulse photocoagulation, sparing (we hope) the photoreceptors. The number of burns required was possibly more than would be expected using the argon laser, although recent studies have indicated that many patients are currently undertreated. ${ }^{12}$ The fact that the burns were not visible clinically made treatment slightly more difficult to perform.

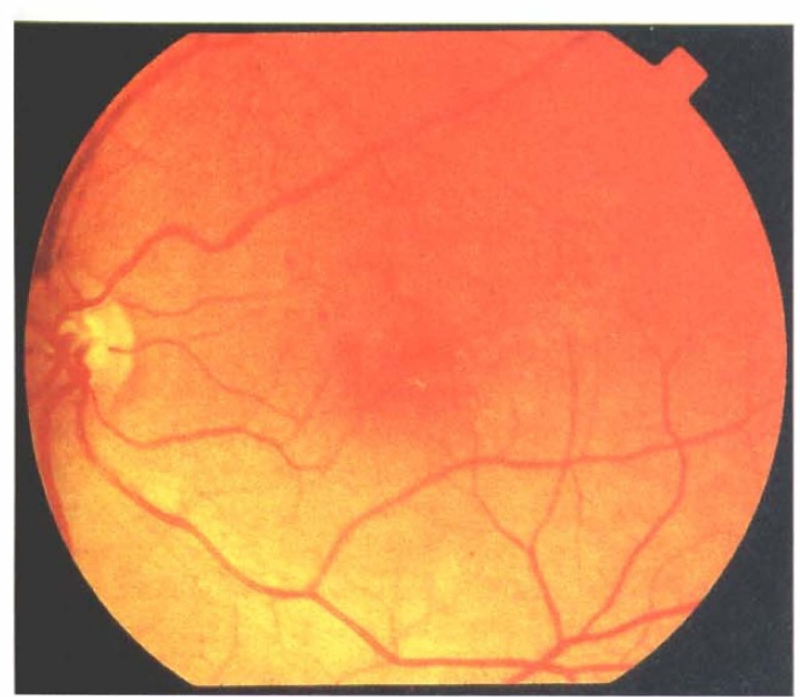

(a)

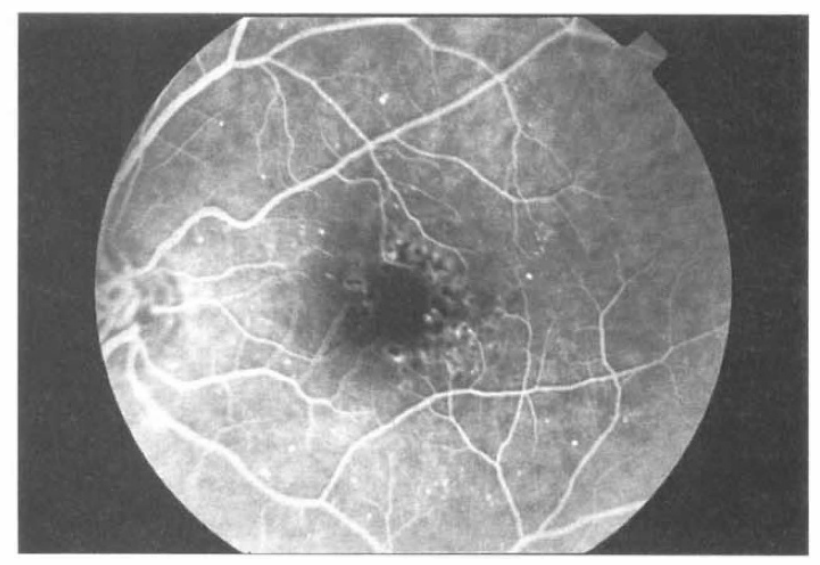

(b)

Fig. 4. (a) Patient 3. Fundus photograph of the left eye 3 months after treatment for macular oedema. There is faint, patchy hypo-pigmentation at the level of the RPE. (b) Fluorescein angiogram of the same eye. 
We are currently recruiting patients to a randomised controlled study comparing the effects of conventional focal and panretinal argon green laser therapy with micropulsed diode laser treatment on visual fields and contrast sensitivity. Preliminary data suggest that the photoreceptor and inner retinal layers are preserved during treatment with the MicroPulse diode laser.

\section{Conclusion}

Patients tolerate treatment with the MicroPulse diode laser very well. It would appear that it is as efficient as the argon laser in the treatment of macular oedema and less disruptive; in many cases there was no visible evidence of treatment months later. Panretinal photocoagulation is also effective, with the majority of patients responding well. Further studies are needed to assess this effect in a larger group of patients. There were no complications or adverse effects noted in the patients treated with this form of laser.

\section{References}

1. Pearson AR, Tanner V, Krightley SJ, Casswell AG. What effect does laser photocoagulation have on driving visual fields in diabetics? Eye 1998;12:64-8.

2. Ulbig M, Arden G, Hamilton P. Color contrast sensitivity and pattern electroretinographic findings after diode and argon laser photocoagulation in diabetic retinopathy. Am J Ophthalmol 1994;117:583-8.
3. Chong LP, Soriano D, Ramos AR. Sublethal laser damage to the retinal pigment epithelium by micro-pulse diode laser in primate eye [abstract]. Invest Ophthalmol Vis Sci 1996;37:S694.

4. Friberg TR, Venketesh S. Alteration of pulse configuration affects the pain response during diode laser photocoagulation. Lasers Surg Med 1995;16:380-3.

5. Early Treatment Diabetic Retinopathy Study Research Group. Early photocoagulation for diabetic retinopathy. ETDRS report 9. Ophthalmology 1991;98:757-85.

6. Resser F, Fleischmann J, Williams GA, Goldmann A. Efficacy of argon laser photocoagulation in the treatment of circinate diabetic retinopathy. Am J Ophthalmol 1981;92:762-7.

7. Ulbig MRW, McHugh DA, Hamilton AMP. Diode laser photocoagulation for diabetic macular oedema. $\mathrm{Br} \mathrm{J}$ Ophthalmol 1995;79:318-21.

8. Friberg TR, Karatza EC. The treatment of macular disease using a micropulsed and continuous wave $810 \mathrm{~nm}$ diode laser. Ophthalmology 1997;104:2030-8.

9. Sato Y, Berkowitz BA, Wilson CA, De Juan E. Blood-retinal barrier breakdown caused by diode vs argon laser photocoagulation. Arch Ophthalmol 1992;110:277-81.

10. Kim JE, Pulido JS, Burkel JM. The actin cytoskeleton during tissue repair in situ after micropulse laser ablation of the RPE monolayer. Invest Ophthalmol Vis Sci 1995;36(Suppl):3842.

11. Ruskovic D, Boulton M, Ulbig MW, Watt M, McHugh DA, Marshall J. The effect of micropulsed diode laser on human RPE in vivo and in vitro [abstract]. Invest Ophthalmol Vis Sci (ARVO Suppl) 1997;38:3483, S754.

12. Cordeiro MF, Stanford MR, Phillips PM, Schilling JS. Relationship of diabetic microvascular complications to outcome in panretinal photocoagulation treatment of proliferative diabetic retinopathy. Eye 1997;11:531-6.

13. Boutacoff TA, Buzawa D, Dorin G, Kelsoe W. Diode laser MicroPulse 'minimal impact photocoagulation' (DLMP) [unpublished data]. Iris Medical Instruments, 1997. 